\title{
DESIGN STUDY OF 2-IN-1 LARGE-APERTURE IR DIPOLE (D2) FOR THE LHC LUMINOSITY UPGRADE*
}

\author{
Vadim V. Kashikhin", Alexander V. Zlobin, FERMILAB, Batavia, IL 60510, U.S.A.
}

\section{Abstract}

This paper analyses possible D2 magnet designs for the "dipole-first" option of the LHC luminosity upgrade based on $\mathrm{Nb}_{3} \mathrm{Sn}$ superconductor and compares them in terms of the maximum field, field quality, and Lorentz forces in the coils.

\section{INTRODUCTION}

After LHC operates for several years at nominal parameters it will need an upgrade to higher luminosity. Replacing the low-beta insertions with a higher performance design based on advanced superconducting magnets is a straightforward step in this direction. One of the approaches being considered for the new LHC IRs is a "dipole-first" option with two separation dipoles placed in front of the focusing quadrupoles [1]. It reduces the number of parasitic collisions with respect to the "quadrupole-first" option and allows independent field error correction for each beam.

Most of key magnet designs for the "dipole-first" option have already been studied and reported. Magnetic and mechanical designs of D1 magnet were analyzed. It was demonstrated that the "open midplane" design can meet the requirements in spite of the large heat depositions [2]. 2-in-1 quadrupole magnets have also been studied. It was shown that the magnets can operate at the nominal LHC IR gradient in 100-mm apertures with the nominal LHC beam separation and good field quality [3].

This paper focuses on the design studies of the 2-in-1 separation dipole (D2) located between D1 and the 2-in-1 quadrupole magnets.

\section{MAGNET DESIGNS}

The D2 magnet should produce magnetic field of 14.1 $\mathrm{T}$ on the length of $10 \mathrm{~m}$ [1]. The magnet apertures have to provide the same field polarity for two counter-rotating beams that implies negative coupling (or mutual inductance) between the apertures. Apart from the necessity to adjust the coil geometry to compensate for the coupling, it creates an additional design challenge since a considerable part of the flux returns through the iron yoke, unlike the case of opposite polarity, when about half of the flux returns through the second aperture.

The size of magnet dynamic aperture is determined by the beam envelope at the magnet location. In this study it was assumed that the D2 magnet apertures are parallel, therefore the dynamic aperture must also account for the beam deflection under the dipole field. Beam optics studies have shown that the round beam envelope at the non-IP end of D2 is $38 \mathrm{~mm}$ that shrinks to $29 \mathrm{~mm}$ at the IP

\footnotetext{
*Work was supported by the US Department of Energy "vadim@fnal.gov
}

end [4]. The maximum beam deflection off the magnet axis is $36 \mathrm{~mm}$ that defines the dynamic aperture of 69.5 $\mathrm{mm}$. In addition to the dynamic aperture, the magnet physical aperture should include the helium channel and absorber that for the heat depositions at the luminosity of $10^{35} \mathrm{~cm}^{-2} \mathrm{~s}^{-1}$ [5] brings the magnet aperture to $\sim 100 \mathrm{~mm}$.

Two different design concepts were considered: one with remote "warm" iron yoke and another one with closer "cold" iron yoke. Both designs are based on fourlayer coil geometry that opens an opportunity for cable grading. However, the negative aperture coupling results in the peak field point located in the outermost layer region adjacent to the second aperture that requires an inverse coil grading to maximize the efficiency with wider cable in the outer layers and narrower cable in the inner layers. The cable parameters for the inner and outer layers are listed in Table 1.

\section{Warm yoke design}

In the warm yoke design, the coils were placed inside a common cylindrical iron yoke, sufficiently remote to accommodate a cryostat. The magnetic coupling between the coils and yoke asymmetry with respect to each coil requires the appropriate asymmetry in the coil geometry to obtain a good geometrical field quality. The coil and yoke geometry were optimized by ROXIE code [6]. Due to the large yoke saturation effect even in the warm yoke design it was necessary to introduce holes in the yoke for better control of the field quality.

Fig. 1 shows the cross-section of optimized D2 coil inside the warm yoke. Unlike the 2-in-1 quadrupole magnet, also using asymmetric coils [3], the coil crosssection requires the same number of turns per layer in each quadrant. However, the turn breakdown between blocks of each layer does not have to be the same for the left and right halves of the coil giving an additional freedom during the optimization.

The warm yoke is distanced from the coils by $\sim 160$ $\mathrm{mm}$ in the midplane. That space is sufficient for the coil support structure and cryostat components including thermal shield, cold-mass supports and vacuum vessel. However, it does not result in a particularly compact magnet design since the yoke has to be relatively thick to limit the field harmonic variations within several units.

Table 1: Cable parameters.

\begin{tabular}{|l|c|c|c|}
\hline \multirow{2}{*}{ Parameter } & \multirow{2}{*}{ Unit } & \multicolumn{2}{|c|}{ Layers } \\
\cline { 3 - 4 } & & Inner & Outer \\
\hline Number of strands & & 17 & 23 \\
\hline Strand diameter & $\mathrm{mm}$ & \multicolumn{2}{|c|}{1.000} \\
\hline Cable inner thickness (bare) & $\mathrm{mm}$ & 1.685 & 1.650 \\
\hline Cable outer thickness (bare) & $\mathrm{mm}$ & 1.881 & 1.915 \\
\hline Cable width (bare) & $\mathrm{mm}$ & 8.627 & 11.694 \\
\hline Copper to non-copper ratio & & \multicolumn{2}{|c|}{1.2} \\
\hline Insulation thickness & $\mathrm{mm}$ & \multicolumn{2}{|c|}{0.18} \\
\hline
\end{tabular}


a)
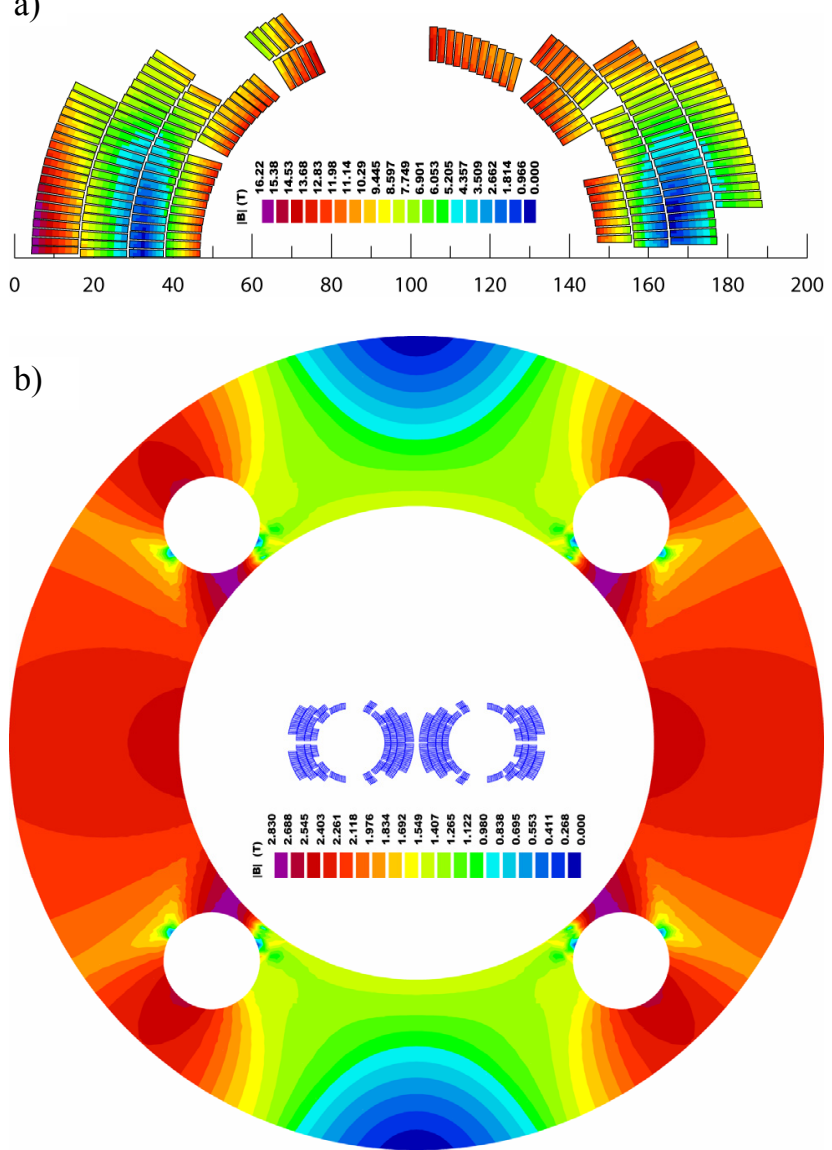

Figure 1: Coil $^{\text {a) }}$ and warm yoke ${ }^{\text {b) }}$ cross-section with magnetic flux density at quench current.

\section{Cold yoke design}

In the cold yoke design, the iron yoke was placed closer to the coils. Due to a complicated yoke shape required in this case, there was no analytical solution available at low fields. The coil and yoke cross-sections were simultaneously optimized for a good geometrical field quality and low yoke saturation effect using ROXIE.

The optimized coil and yoke cross-sections for the cold yoke design are shown in Fig. 2. Due to the small distance between the coils, it was impossible to shield the coils magnetically from each other in this design also, and the coils have to be asymmetric.

\section{MAGNET PARAMETERS}

Calculated parameters of the warm and cold yoke double-aperture dipoles are summarized in Table 2. The parameters responsible for the magnet mechanical performance and quench protection of the two designs are quite close.

In neither case can the target bore field of $14.1 \mathrm{~T}$ be achieved even with the appropriately graded state of the art $\mathrm{Nb}_{3} \mathrm{Sn}$ cables operating at $1.9 \mathrm{~K}$, although the peak field in the coils is close to $16 \mathrm{~T}$. This low efficiency is related to the negative coupling that raises the peak field in the outer layer, far from the useful field region, thus limiting the magnet performance.
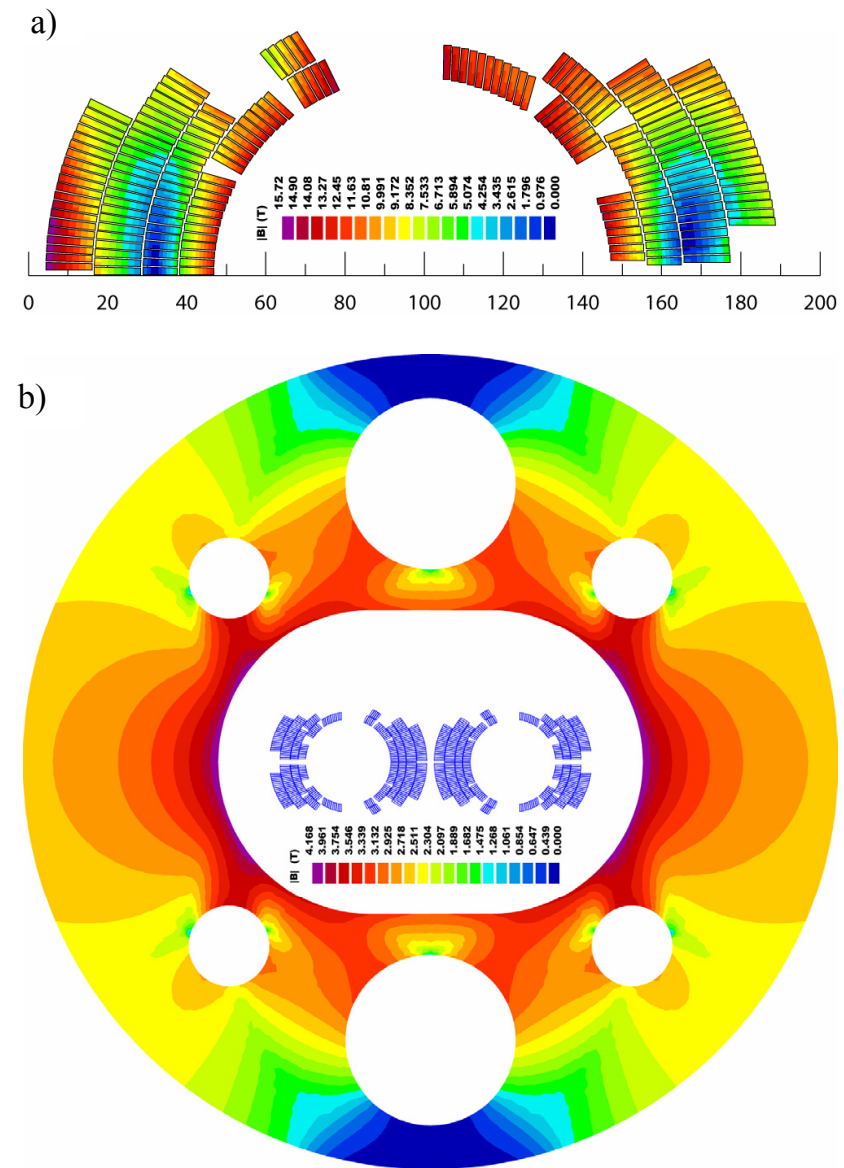

Figure 2: Coil $^{\text {a) }}$ and cold yoke ${ }^{\text {b) }}$ cross-section with magnetic flux density at quench current.

Table 2: Magnet parameters at $\mathrm{T}=1.9 \mathrm{~K}$ for $\mathrm{J}_{\mathrm{c}}(12 \mathrm{~T}$, $4.2 \mathrm{~K})=3000 \mathrm{~A} / \mathrm{mm}^{2}$.

\begin{tabular}{|c|c|c|c|}
\hline \multicolumn{2}{|c|}{ Parameter } & $\begin{array}{l}\text { Warm } \\
\text { yoke }\end{array}$ & $\begin{array}{l}\text { Cold } \\
\text { yoke }\end{array}$ \\
\hline \multicolumn{2}{|c|}{ Aperture diameter, $\mathrm{mm}$} & \multicolumn{2}{|c|}{100} \\
\hline \multicolumn{2}{|c|}{ Number of turns/aperture } & \multicolumn{2}{|c|}{196} \\
\hline \multicolumn{2}{|c|}{ Yoke outer diameter, $\mathrm{mm}$} & 1200 & 960 \\
\hline \multicolumn{2}{|c|}{ Quench field in the bore, $\mathrm{T}$} & 12.65 & 13.19 \\
\hline \multicolumn{2}{|c|}{ Quench current, kA } & 14.09 & 14.18 \\
\hline \multicolumn{2}{|c|}{ Transfer function, $\mathrm{T} / \mathrm{kA}$} & 0.898 & 0.930 \\
\hline \multicolumn{2}{|c|}{ Peak field - inner layers, $\mathrm{T}$} & 14.14 & 14.70 \\
\hline \multicolumn{2}{|c|}{ Peak field - outer layers, $\mathrm{T}$} & 15.72 & 16.09 \\
\hline \multicolumn{2}{|c|}{ Quench margin - inner layers, \% } & 3.00 & 0.00 \\
\hline \multicolumn{2}{|c|}{ Quench margin - outer layers, \% } & 0.00 & 2.23 \\
\hline \multicolumn{2}{|c|}{ Inductance/aperture, $\mathrm{mH} / \mathrm{m}$} & 16.48 & 17.22 \\
\hline \multicolumn{2}{|c|}{ Stored energy/aperture, $\mathrm{kJ} / \mathrm{m}$} & 1636 & 1731 \\
\hline \multirow{2}{*}{$\begin{array}{l}\text { Lorentz forces/ } \\
1^{\text {st }} \text { quadrant }\end{array}$} & $\mathrm{F}_{\mathrm{x}}, \mathrm{MN} / \mathrm{m}$ & 5.57 & 6.28 \\
\hline & $\mathrm{F}_{\mathrm{y}}, \mathrm{MN} / \mathrm{m}$ & -5.08 & -5.15 \\
\hline \multirow{2}{*}{$\begin{array}{l}\text { Lorentz forces/ } \\
2^{\text {nd }} \text { quadrant }\end{array}$} & $\mathrm{F}_{\mathrm{x}}, \mathrm{MN} / \mathrm{m}$ & -0.98 & -1.61 \\
\hline & $\mathrm{F}_{\mathrm{y}}, \mathrm{MN} / \mathrm{m}$ & -3.63 & -3.67 \\
\hline
\end{tabular}

Calculated geometrical field harmonics in the warm and cold yoke designs are reported in Table 3 . Due to the coil and yoke asymmetry, the whole spectrum of normal harmonics is allowed in both designs. The low and high order harmonics were effectively suppressed using wedges and midplane shims. 
Fig. 3 shows the yoke saturation effect in the low order harmonics for the two designs. Variations of the quadrupole and sextupole components in both designs are limited to $\pm 2 \cdot 10^{-4}$ for up to the quench currents.

Table 3: Geometrical harmonics at $25 \mathrm{~mm}$ radius, $10^{-4}$.

\begin{tabular}{|c|c|c|}
\hline \multirow{2}{*}{ Harmonic } & \multicolumn{2}{|c|}{ Magnet design } \\
\cline { 2 - 3 } & Warm yoke & Cold yoke \\
\hline $\mathrm{b}_{2}$ & 0.0000 & -0.0001 \\
\hline $\mathrm{b}_{3}$ & 0.0001 & 0.0001 \\
\hline $\mathrm{b}_{4}$ & 0.0002 & 0.0006 \\
\hline $\mathrm{b}_{5}$ & -0.0027 & 0.0062 \\
\hline $\mathrm{b}_{6}$ & 0.0009 & -0.0038 \\
\hline $\mathrm{b}_{7}$ & -0.0084 & 0.0034 \\
\hline $\mathrm{b}_{8}$ & 0.0606 & 0.0186 \\
\hline $\mathrm{b}_{9}$ & -0.0973 & -0.0973 \\
\hline $\mathrm{b}_{10}$ & 0.0889 & 0.0766 \\
\hline $\mathrm{b}_{11}$ & -0.0676 & -0.0552 \\
\hline
\end{tabular}

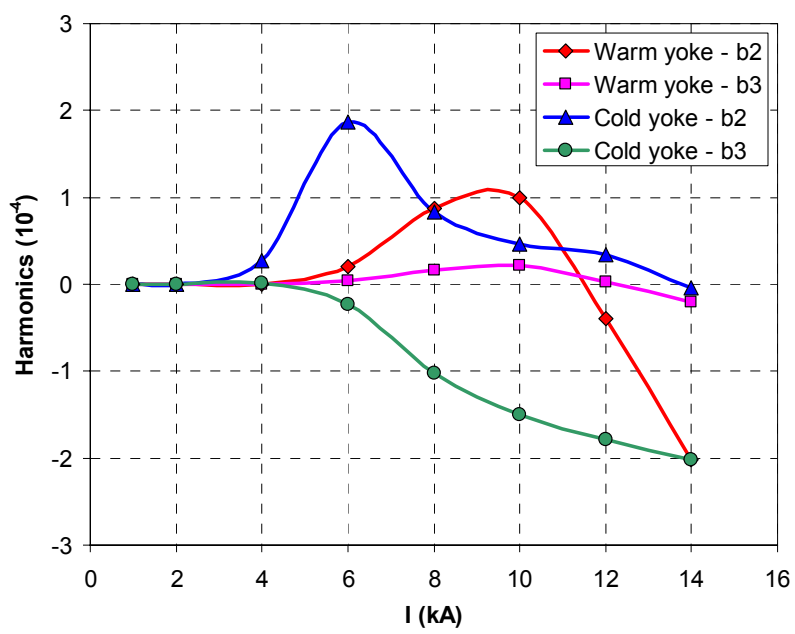

Figure 3: Yoke saturation effect.

\section{DYNAMIC APERTURE}

The D2 dynamic aperture was determined using the same criterion as for the 2-in-1 quadrupoles [3]. Fig. 4 shows the contours of $10^{-4}$ field uniformity in both designs considering the geometrical harmonics up to $b_{18}$. The harmonics that receive the active correction in baseline LHC IR optics $\left(b_{1}-b_{6}\right.$ and $\left.a_{1}-a_{4}\right)$ were assumed to have zero values.

The maximum round beam envelope that fits into the field contours has a diameter of $56 \mathrm{~mm}$ that is $24 \%$ smaller than the required dynamic aperture. So, depending on the beam optics it may be preferable to either increase the magnet physical aperture to $\sim 120 \mathrm{~mm}$ that would reduce the quench bore field to 11-12 $\mathrm{T}$ or accept larger field distortions at the edges of the beam envelope than in the baseline LHC IR optics.

\section{SUMMARY}

Two possible D2 magnet designs based on the warm and cold iron yokes were analyzed. It was shown that these designs have close operating parameters that allows making a design choice based on a preferable mechanical or cryogenic system.

The maximum field that can be reached with the state of the art conductors in $100 \mathrm{~mm}$ apertures is $12.6-13.2 \mathrm{~T}$, depending on the magnet design. An optimistic quench margin of $10 \%$ brings the operating fields down to $11.3-$ $11.9 \mathrm{~T}$ that would require increasing the $\mathrm{D} 2$ magnetic length from $10 \mathrm{~m}$ [1] to $12.5-11.8 \mathrm{~m}$ to keep the same field integral.

The dynamic aperture is practically the same in both designs and smaller than the dynamic aperture required for D2 magnets.

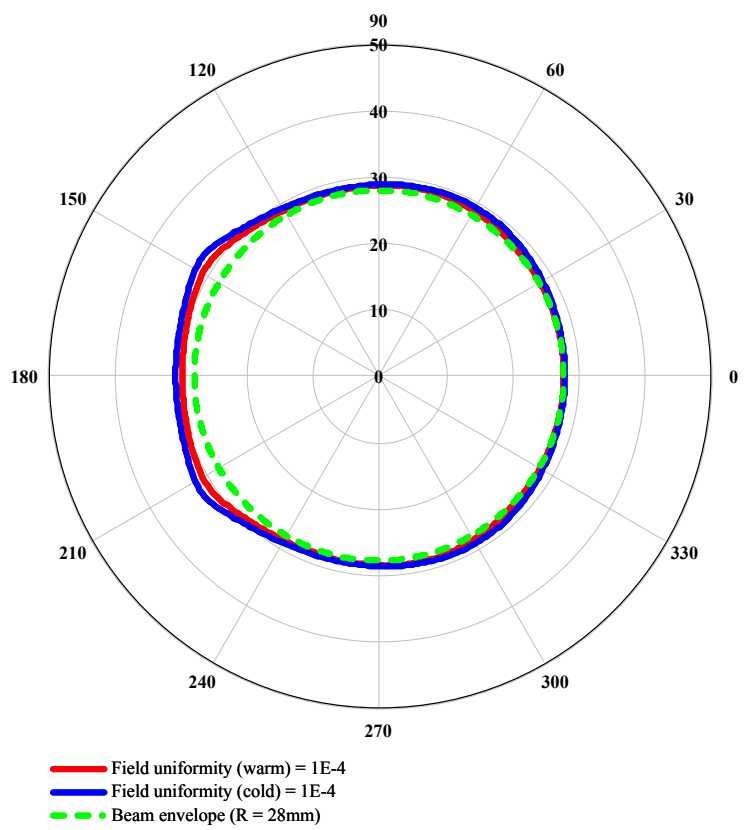

Figure 4: Dynamic aperture of the D2 magnets.

\section{REFERENCES}

[1] J.B. Strait, et al., "Towards a New LHC Interaction Region Design for a Luminosity Upgrade", Proc. 2003 Particle Accelerator Conference, p.42.

[2] M. Anerella, A. Ghosh, R. Gupta, M. Harrison, N. Mokhov, J. Schmalzle, P. Wanderer, "Optimization of Open Midplane Dipole Design for LHC IR Upgrade", Proc. 2005 Particle Accelerator Conference, p.3055.

[3] V.V. Kashikhin, A.V. Zlobin, "Study of 2-in-1 LargeAperture $\mathrm{Nb}_{3} \mathrm{Sn}$ IR Quadrupoles for the LHC Luminocity Upgrade", Proc. 2006 European Particle Accelerator Conference, p.2643.

[4] http://care-hhh.web.cern.ch/carehhh/SuperLHC_IRoptics/IRoptics.html

[5] N. Mokhov, "Energy Deposition Studies of LARP IR Magnets at luminosity of $10^{35} \mathrm{~cm}^{-2} \mathrm{~s}^{-1,}, 2007$ LARP Collaboration Meeting, Fermilab, April 2007.

[6] S. Russenchuck, "A Computer Program for the Design of Superconducting Accelerator Magnets", CERN AT/95-39, LHC Note 354, Sept. 26, 1995. 